\title{
Exploring the Use of Oropharyngeal Cancer Histology to Account for HPV Status in Population-based Studies
}

\author{
UCHECHUKWU C. MEGWALU \\ Department of Otolaryngology-Head and Neck Surgery, Stanford University \\ School of Medicine, Stanford, CA, U.S.A.
}

\begin{abstract}
Aim: To assess whether poorly differentiated tumors and non-keratinizing tumors have similar demographic and clinical characteristics as human papilloma virus (HPV)positive tumors in patients with oropharyngeal cancer. Patients and Methods: The study cohort included patients diagnosed with oropharyngeal squamous cell carcinoma between 2004 and 2012 identified in the Surveillance, Epidemiology, and End Results 18 Database. Results: Poorly differentiated tumors were associated with early $T$ stage (odds ratio $(O R)=1.23$ ), nodal metastasis $(O R=1.66)$ and tonsil fossa origin $(O R=1.22)$. Non-keratinizing tumors were associated with early $T$ stage $(O R=1.23)$, nodal metastasis $(O R=1.66)$ and tonsil fossa origin $(O R=1.22)$. Poorly differentiated tumors were associated with improved overall survival (OS) (hazard ratio $(H R)=0.78$, $p<0.001)$. Non-keratinizing tumors were associated with improved OS $(H R=0.71, p<0.001)$. Conclusion: Histological grade and keratinization may be useful surrogates to adjust for the effects of HPV status in oropharyngeal cancer studies utilizing population-based cancer databases.
\end{abstract}

Oropharyngeal cancer accounts for $10-12 \%$ of all upper aerodigestive tract cancers (1). The incidence of oropharyngeal cancer is rising (2); approximately 14,000 cases were diagnosed in the United States in 2013, accounting for 2,400 deaths (3). The majority of these cancers are squamous cell carcinoma. The major risk factors or oropharyngeal cancer are tobacco, alcohol consumption and human papilloma virus (HPV) infection $(4,5)$. Oropharyngeal cancer is a major cause of mortality with a 5 -year relative survival rate of approximately $50 \%$ (6).

Correspondence to: Uchechukwu C. Megwalu, MD, MPH, Department of Otolaryngology - Head and Neck Surgery, Stanford University School of Medicine, 801 Welch Road, Stanford, CA 94305, U.S.A. Tel: +1 6507236880, Fax: +1 6507258502, e-mail: megwaluu@yahoo.com

Key Words: Oropharynx cancer, head and neck cancer, human papilloma virus, histology, SEER program.
The management of oropharyngeal cancer still remains controversial. Surgery, radiation therapy and chemotherapy are used for single or multimodality therapy. To date, no randomized controlled trial (RCT) has compared the efficacy for effectiveness of surgical versus non-surgical treatment for oropharyngeal squamous cell carcinoma. Prior attempts at conducting RCTs have been hampered by poor patients' accrual (7). As such, well-conducted comparative effectiveness analyses of population-based observational data may provide much needed information on how to most effectively treat these patients. Unfortunately, most population-based cancer databases do not provide information on HPV status, an important prognostic factor in oropharyngeal cancer.

HPV-positive oropharyngeal cancers have distinct demographic and clinicopathological characteristics. They are more common in white male patients (8-11). They most commonly arise in tonsillar tissue $(9,10)$. HPV-positive cancers usually present with smaller primary tumors and high-rate of nodal metastasis $(9,10)$. Most importantly, patients with HPV-positive oropharyngeal cancers have significantly better prognosis than their HPV-negative counterparts (9-11). Histologically, HPV-positive tumors are more likely to be poorly differentiated and non-keratinizing (12-15). Thus, histological grade and degree of keratinization may be a useful surrogate for HPV status in analysis of population-based cancer databases. Surveillance, Epidemiology, and End Results (SEER) database provides information on histologic grade but no information on HPV status. The goal of this study is to assess whether poorly differentiated tumors and non-keratinizing tumors have similar demographic and clinical characteristics as HPVpositive tumors.

\section{Patients and Methods}

Data were extracted from the Surveillance, Epidemiology, and End Results (SEER) 18 Database of the National Cancer Institute, which includes data obtained from 18 population-based cancer registries in the United States. The study cohort included patients diagnosed with oropharyngeal squamous cell carcinoma between 2004 and 2012. The following International Classification of Diseases or 
Oncology codes were included: C01.9 (Base of tongue, NOS), C02.4 (Lingual tonsil), C05.1 (Soft palate, NOS), C05.2 (Uvula), C09.0 (Tonsillar fossa), C09.1 (Tonsillar pillar), C09.8 (Overlapping lesion of tonsil), C09.9 (Tonsil, NOS), C10.0 (Vallecula), C10.2 (Lateral wall of oropharynx), C10.3 (Posterior wall of oropharynx), (C10.8-Overlapping lesion of oropharynx) and C10.9 (Oropharynx, NOS). Exclusion criteria included multiple primary tumors, cases in which race was recorded as "Unknown" and cases in which the mode of therapy was unknown.

Race was recorded in the SEER database as "White"; "Black"; "Other: American Indian, Alaska Native, Asian/Pacific Islander"; or "Unknown". For analysis, race was dichotomized to "Black" and "White/Other". Marital status was grouped as "married" (including common law) or "single" (single-never married, divorced, widowed). Tumor site was grouped as "base of tongue," "soft palate," "tonsil," "pharyngeal wall" and "Other." Unresectable tumor was defined as T4b for N3 tumor. All cases were coded using the American Joint Committee on Cancer (AJCC) staging 6th edition (16).

The SEER computer software (SEER*Stat version 8.3.2; National Cancer Institute, Bethesda, MD, USA; Information Management Services, Inc., Calverton, MD, USA) was used to extract data from the SEER database. The statistical analysis was performed using IBM SPSS version 20 (IBM Corp., Armonk, NY, USA). Unadjusted logistic regression model was used to compare demographic and clinical characteristics by histologic grade and keratinization. Survival analysis was performed using Kaplan-Meier analysis. Cox proportional hazard regression model was used for multivariable analysis. Race, age, sex, marital status, year of diagnosis, site, AJCC stage group, presence of distant metastasis, presence of unresectable tumor, surgical resection of primary site, treatment with neck dissection and radiation therapy were entered a priori into the model. Cases with missing values were excluded from analysis. An estimate was considered statistically significant at $\alpha=0.05$. This study was exempt from review by the Stanford University School of Medicine Institutional Review Board because it was conducted using de-identified public data.

\section{Results}

Clinical characteristics. From 2004 to 2012, the SEER database identified 20,697 patients meeting the inclusion criteria. The patients' characteristics are displayed in Table I. White patients were more likely to have poorly differentiated tumors than other racial groups (odds ratio $(\mathrm{OR})=1.31,95 \%$ confidence interval $(\mathrm{CI})=1.20-1.43$, $p<0.001)$. White patients were also more likely to have nonkeratinizing tumors than other racial groups $(\mathrm{OR}=1.31,95 \%$ $\mathrm{CI}=1.13-1.52, \quad p<0.001)$. Comparisons of clinical characteristics are shown in Table II. Poorly differentiated tumors were more likely to present with early $\mathrm{T}$ stage than well-differentiated tumors $(\mathrm{OR}=1.23)$. Poorly differentiated tumors were more likely to present with nodal metastasis than well-differentiated tumors $(\mathrm{OR}=1.66)$. Poorly differentiated tumors were more likely to occur in the tonsil fossa than well-differentiated tumors $(\mathrm{OR}=1.22)$. Nonkeratinizing tumors were more likely to present with early $\mathrm{T}$ stage than keratinizing tumors $(\mathrm{OR}=1.23)$. Non-keratinizing tumors were more likely to present with nodal metastasis
Table I. Patients' characteristics.

\begin{tabular}{lc}
\hline Variable & \\
\hline Mean Age (SD) & $58.98(10.53)$ \\
Female & $3,617(17.5 \%)$ \\
Race & $17,878(86.4 \%)$ \\
$\quad$ White & $2,151(10.4 \%)$ \\
Black & $668(3.2 \%)$ \\
Other & \\
Grade & $8,025(38.8 \%)$ \\
Poorly differentiated & $7,977(38.5 \%)$ \\
Well-differentiated & $4,695(22.7 \%)$ \\
Grade not recorded & $1,906(9.2 \%)$ \\
Non-keratinizing & \\
Site & $8,439(40.8 \%)$ \\
Tongue base & $835(4.0 \%)$ \\
Soft palate & $9,950(48.1 \%)$ \\
Tonsil & $295(1.4 \%)$ \\
Pharyngeal wall & $1,178(5.7 \%)$ \\
Other & \\
\hline
\end{tabular}

than keratinizing tumors $(\mathrm{OR}=1.66)$. Non-keratinizing tumors were more likely to occur in the tonsil fossa than keratinizing tumors $(\mathrm{OR}=1.22)$.

Survival analysis. On univariable analysis, patients with poorly differentiated tumors had better overall survival (OS) than patients with well-differentiated tumors (5-year OS $=66.3 \%$ vs. $57 \%, p<0.001$ ) (Figure 1). Patients with poorly differentiated tumors had better disease-specific survival (DSS) than patients with well differentiated tumors (5-year DSS $=73.2 \% v s .64 .7 \%, p<0.001$ ). Patients with nonkeratinizing tumors had better OS than patients with keratinizing tumors $(5$-year OS $=73.7 \%$ vs. $59.5 \%, p<0.001)$. Patients with non-keratinizing tumors had better DSS than patients with keratinizing tumors (5-year DSS $=73.2 \% v s$. $64.7 \%, p<0.001$ ).

The results of the multivariable survival analysis are shown in Table III. Poorly differentiated tumors were associated with improved OS (hazard ratio $(\mathrm{HR})=0.78$, $p<0.001)$ ) and DSS (HR=0.75, $p<0.001)$. Non-keratinizing tumors were associated with improved $\mathrm{OS}(\mathrm{HR}=0.71$, $p<0.001)$ and DSS $(\mathrm{HR}=0.68, p<0.001)$.

\section{Discussion}

The results of our study show that poorly differentiated tumors and non-keratinizing tumors have similar characteristics as HPV-positive tumors. Like HPV-positive tumors, poorly differentiated tumors and non-keratinizing tumors are more likely to present with smaller primary tumors (early T stage), are more likely to present with lymph 
Table II. Clinical characteristics by grade and keratinization.

\begin{tabular}{lccc}
\hline Clinical characteristic & Histologic features & Odds ratio $(95 \% \mathrm{CI})$ & $p$-Value \\
\hline Early T stage & Poorly differentiated & $1.23(1.15,1.33)$ & $<0.001$ \\
& Non-keratinizing & $1.52(1.35,1.71)$ & $<0.001$ \\
Nodal metastasis & Poorly differentiated & $1.66(1.53,1.79)$ & $<0.001$ \\
& Non-keratinizing & $1.50(1.30,1.71)$ & $<001$ \\
Tonsil fossa primary & Poorly differentiated & $1.22(1.15,1.30)$ & $<0.001$ \\
& Non-keratinizing & $1.43(1.30,1.57)$ & $<0.001$ \\
\hline
\end{tabular}

CI, Confidence interval.

Table III. Impact of tumor grade and keratinization on survival (multivariable analysis). Each variable was analyzed independently, adjusting for race, age, sex, marital status, year of diagnosis, site, American Joint Committee on Cancer (AJCC) stage group, presence of distant metastasis, presence of unresectable tumor, surgical resection of primary site, treatment with neck dissection and radiation therapy.

\begin{tabular}{lccc}
\hline Survival & Histologic features & Hazard ratio (95\% CI) & $p$-Value \\
\hline Overall survival & Poorly differentiated & $0.78(0.73,0.88)$ & $<0.001$ \\
& Non-keratinizing & $0.71(0.63,0.79)$ & $<0.001$ \\
Disease-specific survival & Poorly differentiated & $0.75(0.69,0.80)$ & $<0.001$ \\
& Non-keratinizing & $0.68(0.60,0.78)$ & $<0.001$ \\
\hline
\end{tabular}

CI, Confidence interval.

node metastasis and are more likely to occur in the tonsil fossa. Similar to HPV-positive tumors, poorly differentiated tumors and non-keratinizing tumors are associated with improved survival.

Tumor HPV status is an important prognostic factor in oropharyngeal cancer. Ang et al. examined the impact of HPV status on survival in 433 patients with advanced oropharyngeal cancer, enrolled in a Radiation Therapy Oncology Group trial comparing two radiotherapy regimens (11). They found that patients with HPV-positive tumors had smaller primary tumors and improved survival. They found that HPV status was an independent prognostic factor for survival on multivariable analysis. Lam et al. examined clinicopathologic features of HPV-associated oropharyngeal cancer in 207 Chinese patients (9). They found that HPV-positive cancers were more likely to occur in the palatine tonsils and with smaller primary tumors. HPV-positive cancers were also associated with increased risk of nodal metastasis but, paradoxically, had improved survival. The similar findings or poorly differentiated and non-keratinizing tumors suggest that histologic grade and keratinization may be useful surrogates to adjust for the effects of HPV status in oropharyngeal cancer studies utilizing population-based cancer databases.

The main strength of our study lies in its large sample size and diverse patients' characteristics. Utilizing the SEER database allows us to analyze a large and diverse population with outstanding quality control. The catchment areas used

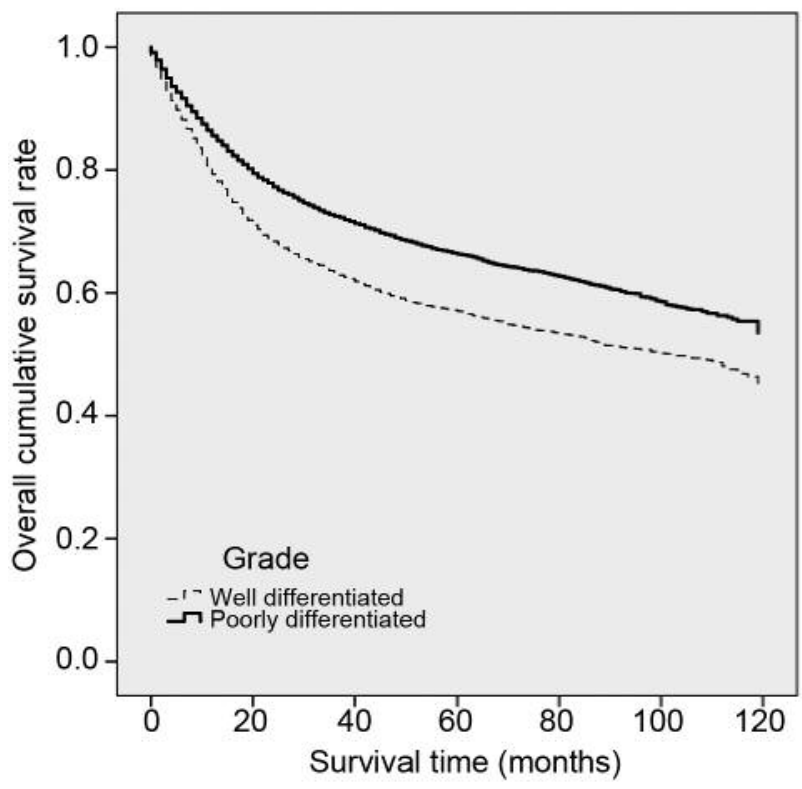

Figure 1. Overall survival as a function of histologic grade. $p<0.001$ by log-rank test.

in the SEER database were selected for their ability to maintain a high-quality cancer reporting system and for demographic characteristics that are representative of the US population as a whole. We limited our analysis to patients 
diagnosed from 2004 to 2012 because the SEER database uses the 6th edition of the AJCC staging criteria only or cases diagnosed after 2003. This study is primarily limited by the retrospective nature of the analysis. In addition, since the SEER database does not provide information on HPV status, direct correlation between HPV status and tumor grade and keratinization cannot be analyzed.

In conclusion, our study suggests that histological grade and keratinization may be useful surrogates to adjust or the effects of HPV status in oropharyngeal cancer studies utilizing population-based cancer databases. Like HPVpositive tumors, poorly differentiated tumors and nonkeratinizing tumors are more likely to present with smaller primary tumors and with lymph node metastasis and are more likely to originate in the tonsil fossa. Similar to HPVpositive tumors, poorly differentiated tumors and nonkeratinizing tumors are associated with improved survival. Further studies are needed to examine the predictive value of histological grade and degree of keratinization or HPV status in population-based cancer databases. The National Cancer Database (NCDB) provides information on HPV status or patients diagnosed after 2008 and may be a useful tool to examine this.

\section{References}

1 D’Souza G, Kreimer AR, Viscidi R, Pawlita M, Fakhry C, Koch WM, Westra WH and Gillison ML: Case-control study of human papillomavirus and oropharyngeal cancer. N Engl J Med 356: 1944-1956, 2007.

2 Auluck A, Hislop G, Bajdik C, Poh C, Zhang L and Rosin M: Trends in oropharyngeal and oral cavity cancer incidence of human papillomavirus (HPV)-related and HPV-unrelated sites in a multicultural population. Cancer 116: 2635-2644, 2010.

3 American Cancer Society: Cancer Facts \& Figures 2015. Atlanta, GA: American Cancer Society; 2015.

4 Blot WJ, McLaughlin JK, Winn DM, Austin DF, Greenberg RS, Preston-Martin S, Bernstein L, Schoenberg JB, Stemhagen A and Fraumeni JF: Smoking and drinking in relation to oral and pharyngeal cancer. Cancer Res 48: 3282-3287, 1988.

5 Herrero R, Castellsagué X, Pawlita M, Lissowska J, Kee F, Balaram P, Rajkumar T, Sridhar H, Rose B, Pintos J, Fernández L, Idris A, Sánchez MJ, Nieto A, Talamini R, Tavani A, Bosch FX, Reidel U, Snijders PJF, Meijer CJLM, Viscidi R, Muñoz N, Franceschi S and IARC Multicenter Oral Cancer Study Group: Human papillomavirus and oral cancer: The International Agency or Research on Cancer multicenter study. J Natl Cancer Inst 95: 1772-1783, 2003.

6 Chen AY and Halpern M: Factors predictive of survival in advanced laryngeal cancer. Arch Otolaryngol Head Neck Surg 133: 1270-1276, 2007.
7 Radiation Therapy Oncology Group: Radiation therapy and cisplatin with or without surgery in treating patients with stage III-IV oropharyngeal cancer. NLM identifier: NCT01953952. ClinicalTrials.gov. Available from: https://clinicaltrials.gov/ct2/ show/NCT01953952?term=Oropharyngeal+cancer\&rank $=10$ [last accessed August 4, 2016].

8 Gooi Z, Chan JYK and Fakhry C: The epidemiology of the human papillomavirus related to oropharyngeal head and neck cancer. The Laryngoscope 126: 894-900, 2016.

9 Lam EWH, Chan JYW, Chan ABW, Ng CS, Lo STH, Lam VSC, Chan MMH, Ngai CM, Vlantis AC, Ma RKH and Chan PKS: Prevalence, clinicopathological characteristics, and outcome of human papillomavirus-associated oropharyngeal cancer in southern chinese patients. Cancer Epidemiol Biomarkers Prev 25: 165-173, 2016.

10 Amini A, Jasem J, Jones BL, Robin TP, McDermott JD, Bhatia S, Raben D, Jimeno A, Bowles DW and Karam SD: Predictors of overall survival in human papillomavirus-associated oropharyngeal cancer using the National Cancer Data Base. Oral Oncol 56: 1-7, 2016.

11 Ang KK, Harris J, Wheeler R, Weber R, Rosenthal DI, NguyenTân PF, Westra WH, Chung CH, Jordan RC, Lu C, Kim H, Axelrod R, Silverman CC, Redmond KP and Gillison ML: Human papillomavirus and survival of patients with oropharyngeal cancer. N Engl J Med 363: 24-35, 2010.

12 Andl T, Kahn T, Pfuhl A, Nicola T, Erber R, Conradt C, Klein W, Helbig M, Dietz A, Weidauer H and Bosch FX: Etiological involvement of oncogenic human papillomavirus in tonsillar squamous cell carcinomas lacking retinoblastoma cell cycle control. Cancer Res 58: 5-12, 1998.

13 Wilczynski SP, Lin BT, Xie Y and Paz IB: Detection of human papillomavirus DNA and oncoprotein overexpression are associated with distinct morphological patterns of tonsillar squamous cell carcinoma. Am J Pathol 152: 145-156, 1998.

14 Klussmann JP, Weissenborn SJ, Wieland U, Dries V, Eckel HE, Pfister HJ and Fuchs PG: Human papillomavirus-positive tonsillar carcinomas: A different tumor entity? Med Microbiol Immunol (Berl) 192: 129-132, 2002.

15 El-Mofty SK and Lu DW: Prevalence of human papillomavirus type 16 DNA in squamous cell carcinoma of the palatine tonsil, and not the oral cavity, in young patients: a distinct clinicopathologic and molecular disease entity. Am J Surg Pathol 27: 1463-1470, 2003.

16 Greene FL, Page DL, Fleming ID, Fritz AG, Balch CM, Haller DG and Morrow M: AJCC cancer staging manual. 6th edition. New York: Springer-Verlag, 2002.
Received August 24, 2016

Revised September 13, 2016

Accepted September 14, 2016 